\title{
artigo
}

Costa, C.R.M.; Rodrigues, A.S.; Araújo, A.C.S.P.; Santos, N.B.; Teixeira, R.C.; Lira, S.C.S.;

Qualidade de vida do idoso ribeirinho da Amazônia

\section{Qualidade de vida do idoso ribeirinho da Amazônia}

\author{
Quality of life of the elderly people from the Amazon \\ Calidad de vida de los mayores gentes de la Amazonia
}

\begin{abstract}
RESUMO
OBJETIVO: avaliar a qualidade de vida de idosos ribeirinhos e urbanos da amazônia. MÉTODO: Foi utilizado questionário World Health Organization Quality of Life - bref em 79 idosos, sendo este 43 ribeirinhos de Cametá- PA e 36 urbanos de Belém- PA, e analisados pela estatística descritiva e teste T de student adotando-se um $\mathrm{p}<0,05$. RESULTADOS: De modo geral, a percepção da qualidade de vida $(p=0,154)$ e a satisfação com a saúde $(p=0,928)$ é a mesma entre idosos ribeirinhos e urbanos. Em análise específica por domínios, ribeirinhos têm melhor qualidade de vida no aspecto psicológico $(p=0,042)$, relações sociais $(p=0,021)$ e meio ambiente $(p=0,001)$, quando comparado aos idosos urbanos. Além disso, em análise mais detalhada por cada domínio os ribeirinhos têm resultados mais satisfatórios em quase 50\% dos itens do questionário. CONCLUSÃO: Idosos ribeirinhos têm qualidade de vida elevada pelos domínios psicológicos, relações sociais e meio ambiente quando comparados aos urbanos.
\end{abstract}

DESCRITORES: Qualidade de Vida; Idosos; Envelhecimento da População; População urbana; População rural.

\section{ABSTRACT}

AIM: This work aimed to evaluate the quality of life of the elderly in the region and in urban Riverside Amazon context. METHOD: The questionnaire was used World Health Organization Quality of Life-bref in 79 seniors, being the region's 43 Riverside municipality of Cametá-PA and 36 in the metropolitan region of Belém-PA, and analyzed by descriptive statistics and student T test by adopting a level of significance of 0.05. RESULTS: In General, the perception of quality of life $(p=0.154)$ and satisfaction with health $(p=0.928)$ is the same between coastal and urban seniors. In specific areas, bordering analysis have better quality of life in the psychological aspect $(p=0.042)$, social relations $(p=0.021)$ and environment $(p=0.001)$, when compared to the elderly. In addition, in more detailed analysis for each domain the bordering have more satisfactory results in almost 50\% of the items in the questionnaire. CONCLUSION: the elderly have high life quality riparian areas, social relations and psychological environment when compared to the elderly.

DESCRIPTORS: Quality of life; Elderly; Ageing of the population; Urban population; The Rural Population.

\section{RESUMEN}

OBJETIVO: Este estudio tuvo como objetivo evaluar la calidad de vida de las personas mayores en las regiones ribereñas y urbanas en el contexto amazónico. MÉTODO: Se utilizó el cuestionario Bref de Calidad de Vida de la Organización Mundial de la Salud en 79 adultos mayores, 43 de la región ribereña del municipio de Cametá-PA y 36 de la región metropolitana de Belém-PA, y se analizó mediante estadística descriptiva y la prueba T de estudiante adoptándola. un nivel de significancia de 0.05. RESULTADOS: En general, la percepción de calidad de vida $(p=0,154)$ y satisfacción con la salud $(p=0,928)$ es la misma entre ancianos ribereños y urbanos. En el análisis específico por dominios, los residentes ribereños tienen mejor calidad de vida en el aspecto psicológico ( $p=0,042)$, relaciones sociales $(p=0,021)$ y medio ambiente $(p=0,001)$, en comparación con los ancianos urbanos. Además, en un análisis más detallado por cada dominio, los habitantes de las riberas tienen resultados más satisfactorios en casi el 50\% de los ítems del cuestionario. CONCLUSION: Los ancianos ribereños tienen una alta calidad de vida debido a los dominios psicológicos, las relaciones sociales y el medio ambiente en comparación con los ancianos urbanos.

DESCRIPTORES: Calidad de Vida; Mayor; Envejecimiento de la Población; Población Urbana; La Población Rural.

RECEBIDO EM: 28/11/2020 APROVADO EM: 08/12/2020

\section{Charles Richardson Moreira da Costa}

Fisioterapeuta, pós-graduado em fisioterapia traumato-ortopédica e fisioterapia desportiva pela Faculdade Integrada da Amazônia.

ORCID: 0000-0001-8377-704X 


\section{Andreia Silva Rodrigues}

Fisioterapeuta, pós-graduada em terapia intensiva neonatal e pediátrica, e pós-graduanda em terapia intensica adulto pela Faculdade Inspirar.

ORCID: 0000-0001-9947-7041

\section{Ana Caroline da Silva Pereira de Araújo}

Fisioterapeuta, pós-graduanda em acupuntura integrativa na Faculdade Inspirar.

ORCID: 0000-0001-8409-2560

\section{Nádia Barreto dos Santos}

Fisioterapeuta, pós-graduada em gerontologia pelo Centro Universitário do Pará e na modalidade residência multiprofissional pela Universidade Federal do Pará, mestre em saúde coletiva pela Universidade Federal do Pará.

ORCID: 0000-0002-4527-1256

\section{Renato da Costa Teixeira}

Fisioterapeuta, doutor em Educação pela PUC-Rio, docente do curso de fisioterapia e do programa de pós-graduação Ensino em Saúde da Amazônia da Universidade do Estado do Pará.

ORCID: 0000-0002-4073-205X

\section{Soanne Chyara Soares Lira}

Fisioterapeuta, mestre em neurociências e biologia celular pela Universidade do Estado do Pará, doutoranda em Ensino em Saúde na Amazônia. Centro Universitário do Pará - CESUPA (Belém/Pará/Brasil). Universidade do Estado do Pará (Belém/ Pará/Brasil) - Grupo de Pesquisa Saúde Funcional e Qualidade de Vida.

ORCID: 0000-0001-5272-9993

\section{INTRODUÇÃO}

C om o aumento da expectativa de vida, a qualidade de vida em idosos é temática amplamente discutida. O termo Qualidade de Vida (QV) vem sendo amplamente discutido na sociedade contemporânea, para melhor compreensão do termo a Organização Mundial de Saúde (OMS) ${ }^{1}$ buscou reunir o que existe de mais significativo na vida das pessoas, as atitudes e situações que mais atuam no seu bem-estar, a partir destes critérios, observou-se que a $\mathrm{QV}$ possui aspectos subjetivos e objetivos, além de possuir um aspecto multidimensional, que envolve o físico, psicológico e social. Por fim, chegou-se ao conceito que QV é a percepção que o indivíduo de sua inserção na vida no contexto da cultura e sistemas de valores nos quais ele vive e em relação aos seus objetivos, expectativas, padrões e preocupações ${ }^{2}$.

Este é um conceito com grande impacto na vida das pessoas, em especial sobre a população idosa, pois incorpora a saúde física de uma pessoa, seu estado psicológico, seu nível de dependência, suas relações sociais, suas crenças e sua relação com características relevantes do ambiente ${ }^{3}$.
Hoje há uma inversão na pirâmide etária, a $\mathrm{OMS}^{4}$ declarou que nas próximas décadas a população mundial com mais de 60 anos vai passar dos atuais 841 milhões para 2 bilhões até 2050. No Brasil, segundo o Instituto Brasileiro de Geografia e Estatística (IBGE), a população de indivíduos com 65 anos ou mais chegou a 7,4\% em 2010. Além disso, de 1940 a 2015, a expectativa de vida no Brasil para ambos os sexos passou de 45,5 anos para 75,5 anos, um aumento de 30 anos $^{5}$.

Em idosos, muito se fala da interferência na QV causada pelos aspectos físicos, como sedentarismo ${ }^{6}$, institucionalização ${ }^{7}$, dos aspectos psicológicos, como a depressão ${ }^{8}$, mas pouco se estuda os aspectos culturais, modo de vida em cenários de metrópole, rurais dentre outros relacionados ao estilo de vida.

Dentro deste contexto, os ribeirinhos, nativos do ambiente amazônico, constituíram seu modo de vida, originando uma população diferenciada pelos seus traços físicos, culturais, alimentares, pelas crenças e formas de organização para o trabalho. Seu perfil regional é caracterizado por sistemas de subsistência dominados pela pesca, caça, cultivo e extração da terra? ${ }^{9}$.
Por sua vez, a população urbana é caracterizada por mais acesso à educação formal, transporte, economia estável, mão de obra industrializada e maior acesso a tecnologias, em outras palavras, a mais condições de vida e saúde no que se concebe na sociedade atual.

$\mathrm{O}$ estudo de Nascimento et $\mathrm{al}^{10}$ mostra que as percepções sobre a população ribeirinha ainda representam uma lacuna para a sociedade e pouco se conhece sobre o processo de envelhecimento vivenciado nesse ambiente que apresenta baixos índices de desenvolvimento humano, além da carência de saúde e amparo social.

Portanto, tratando-se da população idosa ribeirinha e urbana da região amazônica ainda são escassos e limitados os estudos que caracterizam a $\mathrm{QV}$ dentro deste contexto sociocultural. Por isso, esta pesquisa tem como objetivo descrever a qualidade de vida dos idosos ribeirinhos e urbanos no contexto amazônico.

\section{MÉTODO}

Este é um estudo observacional, transversal, quantitativo realizado em idosos nativos residentes em ilhas e em metrópo- 


\section{artigo}

Costa, C.R.M.; Rodrigues, A.S.; Araújo, A.C.S.P.; Santos, N.B.; Teixeira, R.C.; Lira, S.C.S.;

Qualidade de vida do idoso ribeirinho da Amazônia

le do Nordeste da Amazônia Legal. Foram entrevistados 79 idosos, de ambos os sexos, sendo destes, 43 nas regiões fluviais de Cametá e 36 residentes no município de Belém, ambos no Estado do Pará.

A região fluvial avaliada está localizada no baixo Tocantins no nordeste do Estado do Pará, no município de Cametá possuindo uma área territorial de $3.081,367 \mathrm{Km}^{2}$, cujo curso geográfico é composto por 90 ilhas fluviais, com densidade demográfica de 39,23 Hab. $/ \mathrm{km}^{2}$ em 2010 e um índice de desenvolvimento humano de 0.577 no mesmo ano ${ }^{11}$.

Mesmo sendo aposentados, em sua maioria, os idosos residentes nesta região ainda vivem do trabalho artesanal e rural que movimenta a região como o cultivo de açaí, a pescaria de peixes e camarão, além da confecção de paneiros de açaí, os quais servem para armazenar a fruta logo após sua retirada da árvore 9 . Para o contexto da pesquisa foram consultados os idosos residentes na ilha de Jacaré Xingu, afastada do centro urbano e com acesso somente por meio de transporte fluvial.

A população urbana escolhida para compor este trabalho reside no munícipio de Belém e tem uma população estimada para o ano de 2017 de 1.452 .275 habitantes, sendo estes 129.929 idosos com idade igual ou superior há 60 anos, no ano de 2010 possuía densidade demográfica de $1315,27 \mathrm{Hab} . / \mathrm{Km}^{2}$ e índice de desenvolvimento humano de 0,74612. Nesta região a grande maioria dos moradores já se encontram aposentados e alguns ainda possuem trabalhos em suas mais variadas possibilidades, desde artesanato, trabalhos domésticos e até mesmo empregos fixos de boa remuneração.

A pesquisa está em consonância com os princípios éticos do Conselho Nacional de Saúde (Res. CNS 466/12), sendo realizada no período de julho a dezembro de 2016, após a aprovação do Comitê de ética do Centro Universitário do ParáCESUPA, sob o parecer $n^{\circ} 019582 / 2016$ (CAAE: 54215216.7.0000.5169).

Os participantes se deram por conveniência e atenderam aos seguintes critérios de inclusão: possuir idade igual ou superior a 60 anos e residir nas regióes citadas há pelo menos seis meses, que aceitassem assinar o termo de consentimento - TCLE - e em condições de responder ao questionário. Foi realizado o levantamento de dados sociodemográficos de ambos os públicos. Para a coleta de dados foi realizada a aplicação do questionário da World Health Organization Quality of Life - bref (WHOQOL-bref), que avalia a percepção do indivíduo de sua posição na vida, no contexto da cultura e do sistema de valores em que vive e em relação aos seus objetivos, expectativas, padrões e preocupações $^{13}$. O questionário é composto de 26 questões objetivas classificadas nos domínios: físicos, psicológicos, relações sociais e meio ambiente. Foi utilizada análise descritiva dos itens avaliados na escala sendo realizado média dos escores, com a distribuição das variáveis.

Para análise estatística inferencial, foram aplicados testes paramétricos devido a distribuição normal dos dados. Foi utilizado o teste $t$ de Student e estatística descritiva dos escores. Os testes estatísticos foram realizados por meio do software SPSS 10.0 para Windows (Statistical Package for Social Sciences, versão 10.0, 1999), adotando-se nível de significância fixado em $5 \%(\alpha=0,05)$.

\section{RESULTADOS}

Os idosos residentes na região metropolitana de Belém apresentaram média de idade 70,54 anos $( \pm 7,30)$, e os que moram nas ilhas amazônicas apresentam média de 68,11 anos $( \pm 6,65)$. Quanto ao sexo observou-se alta prevalência de idosas urbanas $(68,57 \%)$, já o público da região dos rios amazônicos se mostrou mais homogêneo sendo $51,16 \%$ feminino e $48,84 \%$ masculino como representado na tabela 1 .

A comparação da qualidade de vida está exposta por domínios do WHOQOL-bref (TABELA 2). A percepção da qualidade de vida - PQV ( $\mathrm{p}=0,154)$ e satisfação de saúde $-S S(p=0,928)$, não tiveram diferença significativa entre os grupos.

\section{Tabela 1: Idade e Sexo dos idosos participantes da pesquisa.}

\begin{tabular}{|c|c|c|c|c|c|c|c|c|c|}
\hline \multirow{2}{*}{ Variável } & \multicolumn{4}{|c|}{ Ribeirinhos ( $n=43$ ) } & \multicolumn{4}{|c|}{ Urbano (n = 35) } & \multirow{2}{*}{$P$ - Valor ${ }^{(1)}$} \\
\hline & $\mathrm{n}$ & $\%$ & Média & $\mathrm{DP}$ & $\mathrm{n}$ & $\%$ & Média & DP & \\
\hline \multicolumn{10}{|l|}{ Idade } \\
\hline $60-69$ & 28 & 65,12 & \multirow{3}{*}{68,11} & \multirow{3}{*}{ $\pm 6,65$} & 17 & 48,57 & \multirow{3}{*}{70,54} & \multirow{3}{*}{ $\pm 7,30$} & \multirow{3}{*}{0.3343} \\
\hline 70-79 & 12 & 27,91 & & & 14 & 40,00 & & & \\
\hline 80-89 & 3 & 6,98 & & & 4 & 11,43 & & & \\
\hline \multicolumn{10}{|l|}{ Sexo } \\
\hline Masculino & 21 & 48,84 & \multirow{2}{*}{1,42} & \multirow{2}{*}{ $\pm 0,5$} & 11 & 21,43 & \multirow{2}{*}{1,69} & \multirow{2}{*}{ $\pm 0,47$} & \multirow{2}{*}{0.1181} \\
\hline Feminino & 22 & 51,16 & & & 24 & 68,57 & & & \\
\hline
\end{tabular}

Tabela 2: Teste $t$ de Student para comparação dos escores apresentados pelos idosos ribeirinhos e urbanos aos domínios do Questionário WHOQOLBref, por domínio.

\begin{tabular}{|c|c|c|c|c|c|}
\hline \multirow{2}{*}{ Domínios } & \multicolumn{2}{|c|}{ Ribeirinhos } & \multicolumn{2}{|c|}{ Urbanos } & \multirow{2}{*}{ P-Valor ${ }^{(1)}$} \\
\hline & Média & $\pm \mathrm{DP}$ & Média & $\pm \mathrm{DP}$ & \\
\hline Percepção da qualidade de vida & 3,58 & 0,70 & 3,81 & 0,78 & 0.154 \\
\hline Satisfação com a Saúde & 3,58 & 1,10 & 3,56 & 0,93 & 0.928 \\
\hline Domínio Físico & 3,96 & 0,94 & 3,67 & 0,99 & 0.167 \\
\hline Domínio Psicológico & 4,14 & 1,07 & 3,66 & 1,08 & $0.042^{*}$ \\
\hline
\end{tabular}




$\begin{array}{llllll}\text { Relações Sociais } & 4,14 & 1,08 & 3,60 & 1,05 & 0.021^{*} \\ \text { Meio Ambiente } & 4,18 & 0,98 & 3,47 & 1,02 & 0.001^{* *}\end{array}$

Fonte: Dados resultantes da pesquisa (2017).

(1) Teste T de Student para comparação de médias ( $\mathrm{p}$-valor <0.05).

** Valores Altamente significativos; *Valores Significativos.

$\mathrm{Na}$ estatística dos domínios específicos, o domínio físico - DF ( $\mathrm{p}=0,167)$ os grupos se mostraram iguais estatisticamente. Enquanto os domínios psicológicos - DP $(\mathrm{p}=0,042)$, relação social $-\mathrm{RS}(\mathrm{p}=0,021)$ e meio ambiente - MA $(\mathrm{p}=0,001)$ evidenciaram aumento significativo da qualidade de vida nos ribeirinhos.

Em cada domínio específico foi rea- lizada análise univariada de cada faceta (TABELA 3). Em cada domínio, houve facetas em que os idosos ribeirinhos tiveram diferença significativa ou altamente significativa em relação aos urbanos: no DF, a faceta "mobilidade" $(\mathrm{p}=0,019)$, "sono e repouso" ( $\mathrm{p}=0,000)$ e "atividades de vida cotidiana" ( $\mathrm{p}=0,042)$; no DP, a faceta "pensar, aprender, memória e con- centração" ( $p=0,032)$, "satisfação pessoal" $(p=0,048)$ e "sentimentos negativos" $(0,000)$; nas RS, a faceta "atividade sexual" ( $p=0,002)$; no MA, "ambiente físico" $(p=0,000)$, "oportunidades de recreação e lazer" ( $\mathrm{p}=0,029)$, "condições de moradia" $(\mathrm{p}=0,000), \mathrm{e}$ "transporte" $(\mathrm{p}=0,000)$.

\section{DISCUSSÃO}

Todos vão envelhecer, e dentro desse aspecto a qualidade de vida pode se alterar e redescobrir novos significados a partir da experiência. Em geral, idosos tem

Tabela 3: Estatística descritiva das respostas dos idosos aos domínios do questionário WHOQOL-bref, por faceta.

Domínios

Facetas

Dor e desconforto

Dependência de medicamentos

Energia e fadiga

Domínio Físico

Mobilidade

Sono e repouso

Atividades da vida cotidiana

Capacidade de trabalho

Aproveitar a vida

Sentimentos positivos

Domínio Psicológico

Pensar, aprender, memória e concentração Imagem corporal e aparência

Satisfação pessoal

Sentimentos negativos

Relações pessoais

Relações Sociais$$
\text { Atividade sexual }
$$

Suporte (Apoio) social

Segurança física e proteção

Ambiente físico

Recursos financeiros

Oportunidades de adquirir novas informações e habilidades

Oportunidades de recreação/lazer

Condições de moradia

Cuidados de saúde e sociais: disponibilidade e qualidade

Transporte

Grupos

\begin{tabular}{|c|c|c|c|c|}
\hline \multicolumn{2}{|c|}{ Ribeirinhos } & \multicolumn{2}{|c|}{ Urbanos } & \multirow[t]{2}{*}{ P- Valor ${ }^{11}$} \\
\hline Média & $\pm \mathrm{DP}$ & Média & $\pm \mathrm{DP}$ & \\
\hline 3.605 & 1.417 & 3.861 & 1.046 & 0.371 \\
\hline 2.953 & 1.362 & 2.861 & 1.046 & 0.740 \\
\hline 3.907 & 1.171 & 3.750 & 0.996 & 0.528 \\
\hline 4.442 & 1.119 & 3.806 & 1.238 & $0.019^{*}$ \\
\hline 4.465 & 1.008 & 3.472 & 0.971 & $0.000^{* *}$ \\
\hline 4.349 & 0.783 & 3.972 & 0.910 & $0.042^{*}$ \\
\hline 4.279 & 0.959 & 3.972 & 0.845 & 0.139 \\
\hline 3.791 & 0.959 & 3.583 & 0.937 & 0.338 \\
\hline 4.000 & 0.951 & 4.167 & 0.737 & 0.394 \\
\hline 4.163 & 0.949 & 3.694 & 0.951 & $0.032^{*}$ \\
\hline 4.070 & 1.033 & 3.861 & 0.931 & 0.353 \\
\hline 4.581 & 0.823 & 4.278 & 0.659 & $0.048^{*}$ \\
\hline 3.977 & 0.672 & 2,389 & 1.050 & $0.000^{* *}$ \\
\hline 4.511 & 0.631 & 4.306 & 0.856 & 0.223 \\
\hline 4.488 & 0.798 & 3.833 & 1.000 & $0.002^{* *}$ \\
\hline 4.395 & 0.728 & 4.278 & 0.615 & 0.446 \\
\hline 3.791 & 1.440 & 3.742 & 1.082 & 0.278 \\
\hline 4.233 & 0.922 & 3.306 & 0.856 & $0.000^{* *}$ \\
\hline 2.767 & 0.922 & 3.139 & 1.099 & 0.106 \\
\hline 3.814 & 1.296 & 4.000 & 0.986 & 0.482 \\
\hline 3.698 & 1.489 & 3.028 & 1.108 & $0.029 *$ \\
\hline 4.767 & 0.479 & 4.000 & 1.095 & $0.000^{*}$ \\
\hline 3.558 & 1.315 & 3.306 & 1.305 & 0.396 \\
\hline .256 & 0.759 & 3.500 & 1.082 & $0.000^{* *}$ \\
\hline
\end{tabular}

Fonte: Dados resultantes da pesquisa (2017).

(1) Teste T de Student para comparação de médias ( $p$-valor <0.05).

** Valores Altamente significativos; *Valores Significativos. 
uma qualidade de vida reduzida ${ }^{14,15,16}$ por apresentar mais limitações com comprometimentos físicos, como sarcopenia ${ }^{15}$, psicológicos, tornando-os susceptíveis à depressão ${ }^{7,16}$, mudança no papel social ${ }^{17}$, ou mesmo fatores extrínsecos como as desigualdades sociais ${ }^{14}$ que interferem na percepção da saúde dos mesmos. Nesta pesquisa as alterações à saúde surgiram em todos os idosos, independente do ambiente onde vivem, dado este, ratificado pela SS equivalente.

Como as alterações físicas são inerentes ao processo de envelhecimento ${ }^{18}$, idosos ribeirinhos e urbanos são iguais estatisticamente, tendo mais episódios de dor, dependência de medicamentos, menor energia e menor capacidade de trabalho, achados estes, relacionados ao encerramento da vida profissional, o aparecimento de doenças, a dependência de medicamentos, a mudança no estilo de vida ${ }^{19}$.

De maneira esperada, no DF, o estilo de vida de idosos ribeirinhos favorece a mobilidade, sono e repouso e atividades cotidianas, pois estes têm sua renda pautada na pesca e no cultivo de açaí o que mostra que estão em constante atividade física ao subir em árvores para a retirada do fruto, ao jogar e retirar as redes de pesca dos rios, exigindo grande esforço físico, maior capacidade aeróbica e assim uma maior mobilidade para esses indivíduos ${ }^{20}$. $\mathrm{O}$ melhor controle motor (mobilidade) está relacionado com o ambiente e as tarefas por eles executadas ${ }^{21}$, que reduz o nível de dependência e aumenta a qualidade de vida.

Os ribeirinhos, de maneira geral no DP, têm diferença significativa com os urbanos. Fato este relacionado ao "pensar, aprender, memória e concentração", "satisfação pessoal" e "pensamentos negativos". A capacidade de atenção e concentração dos ribeirinhos para a realização de suas atividades seja na pesca, no cultivo ou até mesmo na confecção artesanal dos utensílios utilizados para a sua subsistência, permanecendo ativos e produtivos dentro do seu contexto social. Como ressalta Pojo et $\mathrm{al}^{22}$ o povo ribeirinho, possui um modo de vida atrelado à natureza e aos recursos naturais e conhece, por experiência, os desafios encontrados

e enfrentados na realidade social e cotidiana onde vive. $\mathrm{O}$ sentimento negativo foi o item que apresentou o escore mais alto na população ribeirinha, e pode estar relacionado com o meio de subsistência incerto,

Como as alteraç̧óes

físicas são inerentes

ao processo de

envelhecimento,

idosos ribeirinhos e

urbanos são iguais

estatisticamente,

tendo mais episódios

de dor, dependência

de medicamentos,

menor energia e

menor capacidade

de trabalho, achados

estes, relacionados

ao encerramento da

vida profissional,

o aparecimento

de doenças, a

dependência de

medicamentos, a

mudança no estilo

de vida. pois depende de fases de plantio e reprodução que varia com as estações, e as condições de trabalho em que o "provedor" passa dias longe da família, sem notícias, para garantir a renda necessária. Para um idoso, pequenas preocupações podem tomar grande proporções $^{23}$.

$\mathrm{Na}$ RS geral, os ribeirinhos apresentaram melhor QV. Este resultado pode estar ligado ao maior de amparo familiar no ribeirinho, que geralmente, convive em um espaço físico próximo, até três gerações? Simeão et $\mathrm{al}^{7}$ ressalta que o convívio com os familiares é essencial para a manutenção da QV do idoso, pois lhes traz segurança e sentimento de amor/reconhecimento, além de reduzir os sentimentos negativos e de solidão ${ }^{10,23}$. O item "atividade sexual" foi significativa nos ribeirinhos podendo ser justificado pelo fato de que os idosos apresentam seu comportamento sexual influenciado pela cultura, religião e educação, ressalta-se que a atividade sexual na terceira idade não pode ser visto como algo incompatível, pois está presente em todos os estágios do desenvolvimento humano e pode ser manifestada em atos de carinhos, companheirismo e estar ao lado do parceiro. ${ }^{2425}$

$\mathrm{O}$ ambiente do idoso pode favorecer a QV26 e o domínio MA foi o mais significativo dentre todos os outros domínios, sendo melhor para o ribeirinho. Quanto ao ambiente físico a vida no interior é mais pacata e tranquila, com menor poluição sonora e visual, ao passo que nas grandes cidades a população fica à mercê da falta de segurança e consequente violência, além de uma vida agitada e estressante. Há maior "oportunidades de lazer", principalmente pela visita a vizinhos e parentes que residem nas proximidades, assim como, participação em eventos comunitários como exemplo de jogos de carta, futebol e religiosos ${ }^{27}$. Embora as condições de moradia não sejam ideais do ponto de vista sanitário, sendo a maioria das casas palafitas, em cima dos rios, os idosos de uma maneira geral têm apego pelo que constrói, muitos têm o pensamento que na casa "nascemos, crescemos e morremos, nela o passado o presente e o futuro dão a casa 
o dinamismo diferente" e assim, estando mais satisfeito ${ }^{28,29}$.

A população ribeirinha estudada tem 0 rio como única conexão entre os lares, escolas, mercados e centros sociais e para se deslocar os residentes utilizam como principal meio de transporte o barco. Com diferentes tamanhos e formatos, estas embarcações servem não só de meio para transporte, mas até mesmo para a venda do cultivo, da pesca e do artesanato que garantem a sua subsistência ${ }^{20}$. Os idosos na cidade geralmente encontram dificuldades para locomoção a grandes distâncias, pois para a maioria o principal meio de transporte é coletivo e estes não são adaptados para este público, enfrentando dificuldades como: altura do degrau, dificuldade ao entrar e sair dos ônibus ${ }^{30}$. Diferente dos ribeirinhos que possuem como único meio de transporte embarcações de portes variados, de uso geralmente familiar.

\section{CONCLUSÃO}

Por meio deste estudo, se identificou que os idosos ribeirinhos apresentaram qualidade de vida geral igual aos urbanos, mas que pelos domínios tiveram melhor resultado no aspecto psicológico, relações sociais e meio ambiente. Estes dados apontam para as vantagens de habitar em locais interioranos, com estilo de vida nativo amazônico, e cultura peculiar que sobrepõem as condições de saúde precárias, economia dependente da safra e ambiente isolado do centro urbano. Por isso evidencia-se que o processo de envelhecimento é igual em ambos os grupos, porém, o viver do idoso ribeirinho é melhor do que o urbano sendo capaz de proporcionar uma qualidade de vida satisfatória. É importante ressaltar que mais estudos devem ser realizados considerando as especificidades e diversidades da população ribeirinha, contribuindo para a promoção da qualidade de vida de idosos.

\section{REFERÊNCIAS}

1. OMS. The World Health Organizations Quality od Life Assessment (WHOQOL): position paper from the World Health Organization. Social Science and medicine. [periodico da internet]. 1995 [acessado em 2020 Nov 01]; 41(10); [cerca de 18 p.]. Disponivel em: https://doi.org/10.1016/0277-9536(95)00112-K

2. Minayo MCS, Buss, PM, Hartz ZMA. Qualidade de vida e saúde: um debate necessário. Revista Ciência e Saúde Coletiva. 2000 [acessado em 2020 Nov 01];5(1):[cerca de 10p.]. Disponível em: http://dx.doi.org/10.1590/S1413-81232000000100002

3. Geib LTC. Determinantes Sociais Da Saúde Do Idoso. Revista Ciência e Saúde Coletiva [periódico na internet]. 2012 [acessado em 2020 Out 01];17(1):[cerca de 10 p.] Disponivel em: http://dx. doi.org/10.1590/S1413-81232012000100015

4. Organização Mundial da Saúde. Relatório mundial de Envelhecimento e saúde. Revista Brasileira de Geriatria e Gerontologia [periódico na internet]. 2015 [acessado em 2020 Nov 01]. Disponivel em: http://sbgg.org.br/wp-content/uploads/2015/10/ OMS-ENVELHECIMENTO-2015-port.pdf

5. Instituto Brasileiro de Geografia e Estatística (IBGE) [Internet]. IBGE Notícias. 2017. [acessado em 2020 Out 05]. Disponivel em: https://agenciadenoticias.ibge.gov.br/agencia-noticias/2013-agencia-de-noticias/releases/9490-em2015-esperanca-de-vida-ao-nascer-era-de-75-5-anos.html

6. Ferreti F, Beskow GCT, Slaviero RC; Ribeiro CG. Análise da qualidade de vida em idosos praticantes e não praticantes de exercício físico regular. Revista Estudo Interdisciplinar do Envelhecimento, [periódico da internet]. 2015. [Acessado em 2020
Nov 01];20(3):[cerca de 15 p.] Disponivel em: http://seer.ufrgs. br/index.php/RevEnvelhecer/article/view/41384

7. Scherrer Júnior Gerson, Okuno Meiry Fernanda Pinto, Oliveira Leticia Meazzini de, Barbosa Dulce Aparecida, Alonso Angélica Castilho, Fram Dayana Souza et al . Qualidade de vida de idosos institucionalizados com e sem sinais de depressão. Rev. Bras. Enferm. [Internet]. 2019 [cited 2020 Nov 20] ; 72( Suppl 2 ): 127-133. Available from: http://www.scielo.br/scielo. php?script=sci_arttext\&pid=S0034-71672019000800127\&Ing=en. Epub Dec 05, 2019. https://doi.org/10.1590/00347167-2018-0316.

8. Corrêa, Mariana Lima etal. Depressão em idosos de uma região rural do Sul do Brasil. Ciência \& Saúde Coletiva [online]. v. 25, n. 6 [Acessado em 2020 Nov 01] , pp. 2083-2092. Disponível em: <https://doi. org/10.1590/1413-81232020256.18392018>. ISSN1678-4561. https://doi.org/10.1590/1413-81232020256.18392018.

9. Reis DC, Araújo MEC, Santos SSL, Silva SSC, Pontes FAR. Araraiana e combu: um estudo comparativo de dois contextos ribeirinhos amazônicos. Resvista Temas em Psicologia, [periódicos na internet]. 2012. [Acessado em 2020 Set 06];20(2):[cerca de 10 p.]. Disponível em: 10.9788/TP2012.2-11

10. Nascimento RG, Cardoso RO, Santos ZNL, Pinto DS, Magalhães CMC. Percepção de idosos ribeirinhos amazônicos sobre o processo de envelhecimento: o saber empírico que vem dos rios. Revista Brasileira de Geriatria e Gerontologia, [periódicos na internet]. 2016. [Acessado em 2020 Nov 01];19(2):[cerca de 12 p.]. Disponivel em: http://dx.doi.org/10.1590/180998232016019.150121 


\section{REFERÊNCIAS}

11. Instituto Brasileiro de Geografia e Estatística (IBGE) [Internet]. IBGE Cidades. 2017 [acessado em 2017 Nov]. Disponível em: https://cidades.ibge.gov.br/painel/painel.php?codmun $=150210$

12. Instituto Brasileiro de Geografia e Estatística (IBGE) [Internet]. IBGE Cidades. 2017. [acessado em 2017 Nov]. Disponível em: https://cidades.ibge.gov.br/painel/painel.php?lang=\&codmun=150140\&search=par\%E1|belem

13. Fleck MPA, Lousada S, Xavier M, Chachamovich E, Vieira G, Santos L, Pinzon V. Aplicação da versão em português do instrumento de avaliação da qualidade de vida da organização mundial da saúde (WHOQOL-100). Revista saúde pública, [periódico da internet]. 1999. [acessado em 2017 Nov];33(2):[cerca de 9 p.]. Disponível em: http://dx.doi.org/10.1590/S003489101999000200012

14. Paiva, Mariana Mapelli de, Lima, Margareth Guimarães e Barros, Marilisa Berti de Azevedo. Desigualdades sociais do impacto das quedas de idosos na qualidade de vida relacionada à saúde. Ciência \& Saúde Coletiva [online]. v. 25, n. 5 [Acessado 20 Novembro 2020] , pp. 1887-1896. Disponível em: <https:// doi.org/10.1590/1413-81232020255.34102019>. ISSN 16784561. https://doi.org/10.1590/1413-81232020255.34102019.

15. Fipplin LI, Miraglia F, Teixeira VNO, Boniatti MM. Timed Up and Go test no rastreamento da sarcopenia em idosos residentes na comunidade. Revista Brasileira de Geriatria e Gerontologia [periódico na Internet]. 2017 [acessado em 2020 Nov 15];20(4):[cerca de 6 p.]. Disponivel em: http://dx.doi. org/10.1590/1981-22562017020.170086

16. Almeida OLS. Saúde mental do idoso: uma questão de saúde pública. Medicina (Ribeirao Preto) [Internet]. 14 de outubro de 2020 [citado 19 de novembro de 2020];53(3):E1-E3. Disponível em: https://www.revistas.usp.br/rmrp/article/view/174636

17. Silva LM, Silva AO, Tura LFR, Moreira MASP, Nogueira JÁ, Cavalli S. Mudanças e acontecimentos ao longo da vida: um estudo comparativo entre grupos de idosos. Revista Latino-Americana de Enfermagem [periódico da Internet]. 2015 [acessado em 2020 Nov 20];23(1):[cerca de 7 p.]. Disponível em: 10.1590/0104-1169.0144.2518

18. Lima OBA, Lopes MEL, Oliveira AMM, Melo VC. Evidencias da produção científica acerca do envelhecimento: revisão integrativa da Literatura. Revista Brasileira de Ciências da Saúde [periódico na Internet]. 2013 [acessado em 2020 Nov 16];17(2): [cerca de 5 p.]. Disponivel em: 10.4034/RBCS.2013.17.02.15

19. Tavares DMS, Matias TGC, Ferreira PCS, Pegorari MS, Nascimento JS, Paiva MM. Qualidade de vida e autoestima de idosos na comunidade. Revista Ciência e Saúde[periódico na Internet]. 2016 [acessado em 2020 Nov 11];21(11): [cerca de 8 p.]. Disponivel em: 10.1590/1413-812320152111.03032016

20. Silveira FLA, Bassalo TFR. Corpos em equilíbrio: imagens e cotidiano ribeirinho no porto do açaí e na ilha do maracujá, Belém (PA). Revista História, Ciências. Saúde. [Periódico na Internet]. 2012 [acessado em 2020 Nov 11];19(3): [25 p.]. Disponivel em: http://dx.doi.org/10.1590/S0104-59702012000300014.
21. Shumway-cook A, Woollacott M. Controle motor: teoria e aplicações práticas. 3. ed. São Paulo: Manole, 2010.

22. Pojo E. As águas e os ribeirnhos - beirando sua cultura e margeando seus saberes. Revista Margens Interdisciplinar [periódico na Internet]. 2014 [acessado em 2020 Nov 17];8(11). Disponivel em: http://dx.doi.org/10.18542/rmi.v8i11.3249

23. Theeke la, Mallow J. Loneliness And Quality Of Life In Chronically III Rural Older Adults: Findings From a Pilot Study. American Journal Of Nursing [periódico na Internet]. 2013 [acessado 2020 Nov 10];113(9) [cerca de 12p]. Disponivel em: http://10.1097/01. NAJ.0000434169.53750.14

24. Alencar DL, Marques APO, Leal MCC, Vieira, JCM. Exercício da sexualidade em pessoas idosas e os fatores relacionados. Revista Brasileira de Geriatria e Gerontologia [periódico na internet]. 2016 [acessado em 2020 Nov 10]; 19(5): [cerca de 9 p.]. Disponível em: http://dx.doi.org/10.1590/180998232016019.160028

25. Uchôa YS, Costa DCA, Junior IAPS, Silva STSE, Freitas WMTM, Soares SCS. A sexualidade sob o olhar da pessoa idosa. Revista brasileira de geriatria e gerontologia [periódico na internet]. 2016 [acessado em 2020 Nov 10]; 19(6): [cerca de 11 p.]. Disponível em: http://dx.doi.org/10.1590/1981-22562016019.150189

26. Nogueira LRB. Arquitetura vernacular e paisagem amazônica: um caminho na busca pelo habitat poético. Revista da Abordagem Gestáltica [periódico na Internet]. 2016 [acessado em 2020 Nov 16];22(2):[cerca de 10 p.]. disponivel em: http://pepsic.bvsalud.org/scielo.php?script=sci_abstract\&pid =S1809-68672016000200009

27. Gambin G, Molzahn A, Fuhrmann AC, Morais EP, Paskulin LMG. Quality of life of older adults in rural southern Brazil. The International Electronic Jounal of Rural and Remote Health Research, Education Practice and Policy [periódico na Internet]. 2015 [acessado 2020 Nov 12];15(3):[cerca de 12 p.]. Disponível em: https://www.ncbi.nlm.nih.gov/pubmed/26363721

28. Lira TM, Chaves MPSR. Comunidades Ribeirinhas na Amazônia: organização sociocultural e política. Interações Revista Internacional de Desenvolvimento Local [periódico na Internet]. 2016 [acessado 2020 Nov 16];17(1): [cerca de 11p.]. Disponivel em: http://www.interacoes.ucdb.br/article/ view/593/678

29. Demetrio AMV, Barbosa RMSP. Apego, afeto e territorialidade: elos entre o idoso e seu ambiente. Boletim Informativo Unimotrisaúde em Sociogerontologia [periódico na Internet]. 2016 [acessado 2020 Nov 12];7(3):[cerca de 16 p.]. Disponível em: http://www.periodicos.ufam.edu.br/BIUS/article/view/2876

30. Santos MD, Silva MF, Vellaza LA, Pompeu JE. Falta de acessibilidade no transporte público e inadequação de calçadas: efeitos na participação social de pessoas idosas com limitações funcionais. Revista Brasileira de Geriatria e Gerontologia [periódico na Internet]. 2017 [acesso 2020 Nov 13];20(2): [cerca de 14 p.]. Disponível em: http://dx.doi.org/10.1590/198122562017020.160090 